Ophthalmologe 2015 $\cdot 112: 1024$

DOI 10.1007/s00347-015-0171-y

Online publiziert: 17. November 2015

(c) Springer-Verlag Berlin Heidelberg 2015

CrossMark

J. Heichel ${ }^{1}$ F. Bachner ${ }^{1}$ A. Schmidt-Pokrzywniak ${ }^{2} \cdot$ H.-G. Struck ${ }^{1}$ U. Stuhlträger ${ }^{1}$. T. Bredehorn-Mayr ${ }^{1}$

${ }^{1}$ Klinik und Poliklinik für Augenheilkunde, Martin-Luther-Universität Halle-Wittenberg,

Universitätsklinikum Halle (Saale), Halle (Saale), Deutschland

${ }^{2}$ Institut für Medizinische Epidemiologie, Biometrie und Informatik, Medizinische Fakultät,

Martin-Luther-Universität Halle-Wittenberg, Halle (Saale), Deutschland

\title{
Erratum zu: Behandlung kindlicher Tränenwegsstenosen
}

\section{Eine prospektive klinische Kohortenstudie}

Erratum zu:

Ophthalmologe (2015) 112:840-847

DOI 10.1007/s00347-015-0067-x

Die ethischen Richtlinien waren leider nicht korrekt angegeben. Wir bitten, den Fehler zu entschuldigen und die korrigierten ethischen Richtlinien $\mathrm{zu}$ beachten.

\section{Korrespondenzadresse}

\section{Dr. J. Heichel}

Klinik und Poliklinik für Augenheilkunde, Martin-Luther-Universität Halle-Wittenberg, Universitätsklinikum Halle (Saale)

Ernst-Grube-Str. 40, 06120 Halle (Saale)

jens.heichel@uk-halle.de

\section{Einhaltung ethischer Richtlinien}

Interessenkonflikt. J. Heichel, F. Bachner,

A. Schmidt-Pokrzywniak, H.-G. Struck,

U. Stuhlträger und T. Bredehorn-Mayr geben an, dass kein Interessenkonflikt besteht.

Dieser Beitrag enthält Studien an Menschen und Tieren. Eine Zustimmung der Ethikkommission war nicht notwendig. Von allen Patienten liegt eine Einverständniserklärung vor. 